\title{
Tissue Engineering Application of Decellularized Bone for Cranial Defect Repair
}

\author{
Hae Won Shin ${ }^{1,2 *}$, David Nacionales ${ }^{1}$, Patricia Miguez ${ }^{3,4}$, Steven Kim ${ }^{3,4}$, and Dong Joon Lee ${ }^{3}$ \\ ${ }^{1}$ Department of Neurology, School of Medicine, University of North Carolina, USA \\ ${ }^{2}$ Department of Neurosurgery, School of Medicine, University of North Carolina, USA \\ ${ }^{3}$ Oral and Craniofacial Health Sciences Research, School of Dentistry, University of North Carolina, USA \\ ${ }^{4}$ Department of Periodontics, School of Dentistry, University of North Carolina, USA
}

*Corresponding author: Hae Won Shin, Department of Neurology and

Neurosurgery, University of North USA.

Received Date: April 23, 2020

Published Date: May 08, 2020

\begin{abstract}
Large cranial defects are often repaired using graft materials that use new bone to protect the brain, one of the most crucial organs in our body. Currently available graft materials for cranial bone reconstruction include autografts, allografts, xenografts, and synthetic allografts. These options have several drawbacks including donor site morbidity, availability, immunogenic reaction, toxicity, etc. Moreover, the current cranial bone graft materials have additional limitations when further cranial surgery is needed. In an attempt to overcome these limitations, various alternative graft options have been engineered using synthetic or biomimetic materials in the form of porous scaffolds to treat large cranial defects.

The use of natural bone derivatives is relatively uncommon compared to scaffolds made of synthetic or biomimetic biomaterials. Recently, decellularized bone has been applied as a potential graft option for the repair of cranial defects with promising results. Advances in tissue engineering and scaffolding technology may enable decellularized bone to be customized as a defect specific scaffold as an approach to effectively regenerate new bone within the defect. In this review, we focus on recent research using tissue-engineering strategies with decellularized bone to repair cranial bone defects
\end{abstract}

Keywords: Decellularized bone; Immunogenic reaction Scaffold, Tissue-engineering

\section{Introduction}

Cranioplasty is defined as the surgical repair of a congenital or acquired defect in the cranium1 and typically uses graft materials to restore functionality and physical appearance. For centuries, surgeons and scientists have tried to find a better graft material in cranioplasty from xenografts to alloplastic materials. To date, autologous bone grafts have been preferred because of biocompatibility, durability, accessibility and less likelihood of host rejection [1-3]. Although these work well in small to medium cranial defect repairs, these have increased failure risk in large bone defects [3]. In addition, the adequate supply and morbidity at the donor site is a significant limitation of autologous bone grafts. Also, primary cranioplasty failure may lead to partial resorption of the bone and implant detachment up to $22 \%$ of adult patients [3] Thus, alternative alloplastic graft options have been studied and developed.
The most commonly studied and used grafts include PMMA (polymethyl methacrylate), HA(hydroxyapatite), titanium, and PEEK (polyether ether ketone). Table 1 gives a comparison of alloplastic graft options and autologous grafts. HA appears to have the benefits of osteointergration and osteoconductivity but is more brittle and carries an increased risk of fracture. Titanium appears to be strong but causes radiographic artifacts due to the lack of radioluncency. Also, the high conductivity of heat and cold conduction can be problematic. PMMA has good mechanical resistance but high rates of infection. PEEK has high local complication rate and lacks osteointergration and osteoconductivity. Among all of available graft options, the overall complication rate is $9-36 \%$ and $2-10 \%$ of grafts eventually require removal [4]. In addition, the current graft options have significant limitations when a secondary operation at the graft site is required. 
For example, when a patient with prior cranioplasty requires craniotomy or craniectomy due to trauma, tumor, or epilepsy surgery, the second operation is significantly limited. The approach to stereo-EEG (sEEG) implants or responsive neurostimulation therapy (RNS) during epilepsy surgery would be altered when there are previous bone defects or prior graft because of different mechanical resistance, osteoconductivity and osteointergration. Therefore, the development of grafts with improved physical and

Table 1: Comparison among cranial bone grafting options [3]. mechanical characteristics which ideally can regenerate natural bone over time is crucial. The ideal grafts should be biologically inert, promote osteointergration and osteoconductivity, resistant to mechanical failure/fracture, radioluncent, and carry a low risk of infection. Also, these grafts should be readily available even in large bone defect, easy to manipulate and fabricate, and should eventually be replaced by normal bone [3].

\begin{tabular}{|c|c|c|c|c|c|}
\hline & Autologous Bone & HA & Titanium & PMMA & PEEK \\
\hline Biocompatibility & Very High & High & High & Good & High \\
\hline Osteointergration & Yes & Yes & No & No & No \\
\hline Osteoconductivity & Yes & Yes & No & Limited on edge & No \\
\hline Mechanical Resistance & High & Moderate & Very High & High & High \\
\hline Fracture Resistance & Yes & No(first 12 months) & Yes & Yes & Yes \\
\hline Infection Risk & Moderate & Low-moderate & Low-moderate & High & Moderate \\
\hline Radioluncency & Yes & Yes & No & Yes & Yes \\
\hline
\end{tabular}

Decellularized bone matrix (DecBM) has been proposed as a promising candidate for bone graft material. DecBM can be recellularized with autologous stem cells and can potentially be used to design customized grafts for clinical applications. There would be minimal risk of immune reactions due to the removal of cells and adding autologous stem cells. In addition, the similar biochemical and biophysical characteristics as native bone may result in improved integration with host tissues when compared with synthetic grafts. In previous animal studies, decellularization techniques have enabled the development of DecBM with little cytotoxic effect. The optimal decellularization process to create a suitable environment for mesenchymal stem cell's (MSC) adhesion, proliferation, and osteogenic differentiation in vitro also has been determined [5]. In vivo testing has been performed by preparing DecBM in solid and particle forms. The solid DecBM showed limited cellular migration into the dense bone matrices [6]. Whereas the particles induced local bone formation in a rat calvarial critical sized defect (CSD) model, especially between the particles and surrounding host bone. In that study, MSC seeding was shown to have synergic effect on the bone formation [5]. However, similarly to natural bone grafting products that are currently available in the market, the major downside using solid or particle forms of DecBM was the absence of structural integrity, which limits their efficacy to the treatment of small, non-critical sized defects such as dental bone graft applications [7].

CSD is defined as a defect that can't heal without intervention for preclinical in vivo evaluation [8]. Such large defects require structural support initially while osteogenesis is induced with seeded stem cells and osteoconduction occurs from surrounding host tissue during repair. Scaffolds are considered a crucial component because it provides the physical environment for cellular adhesion, growth, and differentiation to facilitate bone regeneration. An ideal bone scaffold is a 3D porous structure where seeded osteoblasts can attach and secrete mineralized extracellular matrices (ECM) that will eventually form new bone within the pores [9]. Among the various types, natural bone derived grafts with a porous structure is the optimal bone scaffold as it possesses identical biological, biochemical, and physical properties as the ECM of natural bone.

The fabrication of scaffolds using DecBM has the potential to present new graft solutions for future large cranial defect repairs. However, naturally derived DecBM are often more difficult to manufacture compared to synthetic biomaterials. Unlike biomimetic or synthetic biomaterials, current design approaches to fabricate 3D porous scaffolds using DecBM have been unsuccessful [10-12] (Figure 1).

The most prominent method to prepare decellularized bone scaffold is mixing decellularized bone matrix with gel type polymers [13]. However, these types of polymers are limited due to their poor stiffness and mechanical integrity. Additionally, it has been difficult to design porous structures using these materials. There has been an attempt to direct print scaffolds using decellularized bone powder, but in this study, DecBM was only added in a small amounts compared to polycaprolactore (PCL), which was the major synthetic bonding material used [14]. Therefore, new fabrication techniques using mechanically reinforced decellularized bone scaffolds with the creation of pores throughout the scaffold is necessary for the future study. Therefore, finding new methods to increase the crosslinking between decellularized bone particles as well as the increasing mechanical strength are key in fabricating an effective bone scaffold for large cranial defect repairs. 

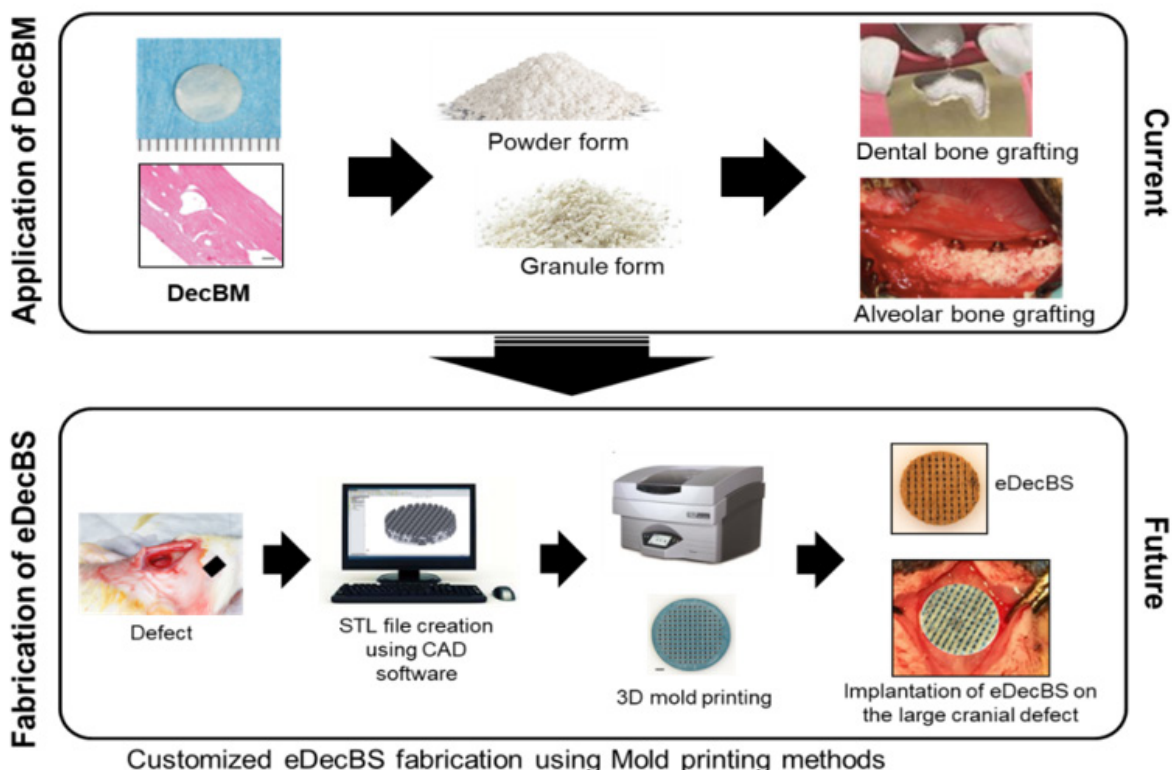

Figure 1: Illustration of current application of DecBM6 in dental and alveolar bone grafting 10,11 and future application using eDecBS on the large size cranial defect. Defect specific 3D porous wax mold can be designed and printed using Solidscape $₫$ Studio 3D printer based on the STL file.12 Then porous eDecBS can be casted in the printed wax mould by chemical reaction process. Surgical implantation of eDecBS into the large cranial defect of rat calvaria model.

\section{Discussion}

For many years, aspirations to regenerate bone using natural grafts have driven significant progress in the field of dentistry and craniofacial surgery. However, the the progress in the regeneration of large cranial defects, which cannot self-repair, using natural grafts has been limited. Although autografts are known as the clinical gold standard, for a massive cranial defects, the supply obtained is often not adequate without the risk of causing severe morbidity at the harvesting site. Structurally, solid DecBM grafts would be an ideal choice for large bone defects as this provides protection at the defect site. However, the regenerative potential is limited due to the compact mineralized nature of the matrix which inhibits the migration of stem cells into the scaffold to induce bone formation. This would cause unstable osteointegration between the graft and host tissue, leading to graft failure. In addition, the decellularized bone graft may have higher degradation than regeneration process. In the absence of osteogenic cells to promote regeneration, the decellularized graft matrices may be degraded by surrounding osteoclasts or enzymatic reactions in the body. Thus, the graft will be weakened over time leading to an increased failure rate.

With recent advances in bioprinting technology, computeraided design (CAD) modeling has been used to fabricate synthetic bone materials. This can enable superior precision in controlling the size and internal environment of bone scaffolds, including enhanced control of the size, connectivity, dimensions, and shape of the pores [15]. Due to the fast-chemical reaction time of the pozzolanic reaction and polymerization of dopamine, the traditional direct 3D printing of the scaffold is not feasible. The 3D mold printing technique has the potential to account for the fast reaction times and successful fabrication of engineered decellularized bone scaffold (eDecBS) that achieves a custom fit (defect specific) design with interconnected pores [16]. eDecBS would meet the criteria of an ideal bone graft and promote bone regeneration through favorable interactions with the seeded stem cells. Additionally, eDecBS would have similar morphological and biomechanical properties as natural bone grafts. By maintaining similar biochemical and biophysical characteristics as native bone, eDecBS can better serve as an osteoconductive matrix with an osteoinductive potential imparted by the retained growth factors and natural bone matrices.

\section{Conclusion}

Although naturally derived bone graft biomaterials likeallografts and xenografts have been utilized for a long time, their application in large defects were limited due to availability and rejection issues. In this review, we briefly discussed decellularization of bone tissue and its future application for large cranial defect repairs. Here, we propose a method to develop the engineered decellularized bone scaffold (eDecBS), which are 3D printed porous scaffolds made of decellularized bone matrix to serve as large bone grafts. The eDecBS seeded with stem cells can be evaluated for its osteogenic potential in repairing large cranial defects in animal models such as rat CSD on cranium models. We anticipate that eDecBS can serve as an excellent candidate for repairing large bone defects.

\section{Acknowledgement}

None.

\section{Conflict of Interest}

The authors declare that they have no conflicts of interest. 


\section{References}

1. Shah AM, Jung H, Skirboll S (2014) Materials used in cranioplasty: a history and analysis. Neurosurg Focus 36(4): E19.

2. Oliver JD, Banuelos J, Abu-Ghname A, Krishna S Vyas, Basel Sharaf (2019) Alloplastic cranioplasty reconstruction: a systematic review comparing outcomes with Titanium Mesh, Polymethyl Methacrylate, Polyehter Ether Ketone, and Norian Implants in 3591 Adult patients. Ann Plast Surg 82: S289-294.

3. Zanotti B, Zingaretti N, Verllicchi A, Massimo Robiony, Alex Alfieri, et al. (2016) Cranioplasty: Review of Materials. J Craniofac Surg 27: 2061 2072.

4. Van de Vifeijken S, Munker, T, Spijker R, et al. (2018) Autologous Bone is Inferior to Alloplastic Cranioplasties: Safety of Autograft and Allograft Materials for Cranioplasties, a Systematic Review. World Neurosurgery 117: 443-52.

5. Lee DJ, Diachina S, Lee YT, Lixing Zhao, Rui Zou, et al. (2016) Decellularized bone matrix grafts for calvaria regeneration. J Tissue Eng

6. Lee DJ, Padilla R, Zhang H, Hu WS, Ko CC (2014) Biological assessment of a calcium silicate incorporated hydroxyapatite-gelatin nanocomposite: a comparison to decellularized bone matrix. Biomed Res Int.

7. Bracey DN, Jinnah AH, Willey JS, Seyler TM, Hutchinson ID, et al. (2019) Investigating the Osteoinductive Potential of a Decellularized Xenograft Bone Substitute. Cells Tissues Organs 207(2): 97-113.

8. Spicer PP, Kretlow JD, Young S, Jansen JA, Kasper FK, (2012) Evaluation of bone regeneration using the rat critical size calvarial defect. Nat Protoc 7(10): 1918-1929.
9. Ghassemi T, Shahroodi A, Ebrahimzadeh MH, Mousavian A, Movaffagh J, et al. (2018) Current Concepts in Scaffolding for Bone Tissue Engineering. Arch Bone JtSurg 6(2): 90-99.

10. Upper Jaw Bone Graft. (2020, March 22). Sinus Lift Los Angeles CA Retrieved.

11. Li H, Zheng J, Zhang S, Yang C, Kwon YD, et al. (2018) Experiment of GBR for repair of peri-implant alveolar defects in beagle dogs. Sci Rep 8(1):16532.

12. Lee DJ, Kwon J, Kim YI, Wang X, Wu TJ, et al. (2019) Effect of pore size in bone regeneration using polydopamine-laced hydroxyapatite collagen calcium silicate scaffolds fabricated by 3D mould printing technology. Orthod Craniofac Res. Suppl 1:127-133.

13. Sawkins MJ, Bowen W, Dhadda P, et al. (2013) Hydrogels derived from demineralized and decellularized bone extracellular matrix. Acta Biomater 9(8): 7865-7873.

14. Hung BP, Naved BA, Nyberg EL, Miguel Dias, Christina A Holmes et al. (2016) Three-Dimensional Printing of Bone Extracellular Matrix for Craniofacial Regeneration. ACS Bio mater Sci Eng 2(10): 1806-1816.

15. Tao O, Kort-Mascort J, Lin Y, et al. (2019) The Applications of 3D Printing for Craniofacial Tissue Engineering. Micromachines (Basel) 10(7): 480.

16. Lee DJ, Kwon J, Kim YI, Wang X, Wu TJ, et al. (2019) Effect of pore size in bone regeneration using polydopamine-laced hydroxyapatite collagen calcium silicate scaffolds fabricated by 3D mould printing technology. Orthod Craniofac Res 22 (Suppl 1): 127-133. 